\title{
Axial mesodermal dysplasia spectrum
}

INSERM

\section{Source}

INSERM. (1999). Orphanet: an online rare disease and orphan drug data base. Axial mesodermal dysplasia spectrum. ORPHA:1834

Axial mesodermal dysplasia spectrum is a rare developmental defect during embryogenesis syndrome characterized by congenital manifestations of both oculoauriculo-vertebral spectrum and caudal regression sequence. Phenotype is highly variable but patients typically present facial dysmorphism (incl. asymmetry, hypertelorism), auricular abnormalities (e.g. preauricular tags, microtia, absence of middle ear ossicles), skeletal malformations (hemivertebrae, hip dislocation, sacral agenesis/dysplasia, talipes equinovarus, flexion deformity of lower limbs), cardiac defects (dextrocardia, septal defects), renal and genitourinary anomalies (such as renal agensis/dysplasia, abnormal external genitalia, cryptorchidia), as well as anal anomalies such as anal atresia and rectovesical fistula. 